\title{
Chaos in Orbits Due to Disk Crossings
}

\author{
C. Hunter \\ Department of Mathematics,Florida State University, \\ Tallahassee FL 32306-4510. Email: hunter@math.fsu.edu
}

\begin{abstract}
We study orbits of halo stars in some simple models of galaxies with disks and halos, to see whether the cumulative effects of the sudden changes in acceleration which occur at disk crossings can induce chaos. We find that they can, though not in all orbits and not in all potentials. Most of the orbits which become chaotic stay relatively close to the disk, and range widely in the radial direction. Heavier disks and increased halo flattening both enhance the extent of the chaos. A limited range of experiments with a three-component model of the Milky Way with an added central bulge finds that many chaotic disk-crossing orbits can be expected in the central regions, and that prolateness of the halo is much more effective than oblateness in generating chaos.
\end{abstract}

Keywords celestial mechanics: stellar dynamics, galaxies: kinematics and dynamics, Galaxy: halo

\section{INTRODUCTION}

Many galaxies have both a flattened disk-like component and much less flattened non-disk components. Normal spirals with their bulges and halos are one example. S0s, which bridge the gap between ellipticals and normal spirals in Hubble's tuning fork diagram [1], are another. More recently it has been recognized that disks can be found in less extreme elliptical galaxies; Bender [2] and Nieto et al [3] have found that many ellipticals can be classified as disky, and that their diskiness is consistent with a disk plus bulge model [4]. The stars which populate the disk can remain forever in the disk, but the gravitational attraction of the disk will cause the orbits of non-disk stars to cross back and forth through the disk. As a star crosses the disk, it will experience an abrupt change in the gravitational force field perpendicular to the disk. This causes an abrupt change in the star's acceleration, though not its velocity, with the result that the curvature of the orbit changes abruptly. Figure 1 illustrates this phenomenon for a razor-thin disk, for which the change is discontinuous.

This paper examines when the cumulative effect of repeated disk crossings leads to chaos. In his efforts to get galactic theorists to recognize the importance of chaos, I recall Henry Kandrup asking me for examples of chaos in axisymmetric systems to help make his point that there is no essential link between triaxiality and chaos. Though I had not thought about the present topic when he first asked, it does provide some clear examples to reinforce his 
point. Figure 2 shows a surface of section for one simple example of a model galaxy with a disk and halo. It shows that chaotic orbits occupy a significant portion of phase space, while there are other parts of phase space in which the orbits are quite regular.

The idea that chaos can be induced by disk crossings is not new, but it has not attracted much attention. One notable exception is that of Ostriker, Spitzer \& Chevalier [5] who discussed the effect that the compressive gravitational shocks, caused by passage through the disk, have on the internal structure of globular clusters. Although we study the simpler matter of individual orbits rather than a whole globular cluster, Ostriker et al. point out that the disruptive effect they study is due to changes in the relative motion of two nearby points of a cluster caused by disk shocking. Orbits of nearby points in a stable cluster are nearby when viewed on the galactic scale. They grow far apart if and when the cluster disrupts. Hence Ostriker et al, like us, are basically concerned with circumstances in which small changes in initial conditions lead to greatly different outcomes. This is well-known to be a hallmark of chaos [6].

The issue of the extent to which orbits in our Galaxy are chaotic was also studied in the 1970s by L. Martinet and co-authors (See [7] and earlier work cited there). They integrated orbits in Schmidt's models of the Galaxy, which have highly flattened disk components, and found extensive regions in which orbits are chaotic. Their surfaces of section are cruder than those we display, which is not in the least surprising because they worked in the era of punched cards and with computers which are primitive by today's standards, and we have not attempted any detailed comparisons with their work.

The present work grew out a study [8] of the Kuzmin-like potentials introduced by Tohline and Voyages [9]. Kuzmin-like potentials provide a simple set of model galaxies with disk and non-disk components. We discuss them and the orbits they support in $\S 2$. We compare and contrast two other simple classes of models, those of Miyamoto \& Nagai [10] and the scalefree logarithmic models of Monet, Richstone \& Schechter [11] and Toomre [12] in $§ 3$. The scalefree models have razor-thin disks, while those of Miyamoto \& Nagai have disks with a non-zero thickenings. Because of their simplicity, Miyamoto \& Nagai disks have often been used to represent the disk in more realistic models of galaxies [13], [14]. Helmi \& White [15] have given an analytical description of how fossils of accreted satellite galaxies

will remain coherent and be detectable in the Milky Way provided that they inhabit regions of phase space in which the motion is essentially integrable. In $\S 4$ we delineate where this is so in some more realistic three-component models of the Galaxy with disk, halo, and bulge. We give our conclusions in $\S 5$

\section{KUZMIN-LIKE MODELS}

\subsection{Kuzmin's Disk}

To understand Kuzmin-like potentials, we first consider Kuzmin's disk [16]. It is a flat and razor-thin density distribution with the remarkable property that the gravitational potential on one of its sides is that due to an image mass on the opposite side, as illustrated in FigURE 
2-5 of Binney \& Tremaine [17]. Its gravitational potential is

$$
\Phi=\frac{-G M}{\sqrt{R^{2}+(a+|z|)^{2}}},
$$

where $z=0$ is the plane of the disk, $R$ measures radial distance from the $z$-axis of symmetry, $M$ is the mass of each image source, and $a$ is their distance from the plane of the disk. The equipotentials are spherical, those in $z>0$ centered on the image mass at $z=-a$ below the disk, and those in $z<0$ centered on the image mass at $z=a$ above the disk.

\subsection{Kuzmin-like Potentials}

The Kuzmin-like potentials of Tohline and Voyages [9] are defined by the more general formula

$$
\Phi=\Phi_{\mathrm{K}}(\xi), \quad \xi=\sqrt{R^{2}+(a+|z|)^{2}} .
$$

Kuzmin's disk is simply the special case of the point mass potential $\Phi_{\mathrm{K}}=-G M / \xi$. The more general Kuzmin-like potentials also have spherical equipotential surfaces centered on opposite sides of the $z=0$ plane. However they are produced by a combination of a thin disk and a volume density. Poisson's equation shows that the general Kuzmin-like potential (2) is generated by the density

$$
\rho=\frac{1}{4 \pi G}\left[\Phi_{\mathrm{K}}^{\prime \prime}(\xi)+\frac{2[1+a \delta(z)]}{\xi} \Phi_{\mathrm{K}}^{\prime}(\xi)\right] .
$$

The Dirac delta function $\delta(z)$ arises from the discontinuity of the derivative of $|z|$, and gives the thin disk. Kuzmin-like potentials therefore arise from a volume density which is stratified on the equipotentials, and a surface density $\Sigma=a \Phi_{\mathrm{K}}^{\prime}(\xi) / 2 \pi G \xi$ on the plane $z=0$. It is this surface density which causes the $z$-component of force to be discontinuous. The volume density vanishes for the special case of Kuzmin's disk, when there is only a surface density

$$
\Sigma(R)=\frac{a M}{2 \pi\left(a^{2}+R^{2}\right)^{3 / 2}} .
$$

The freedom to choose the spherical potential $\Phi_{\mathrm{K}}(\xi)$ allows there to be a considerable variety of Kuzmin-like potentials for modeling galaxies with both disks and halos. However a choice of $\Phi_{\mathrm{K}}(\xi)$ fixes both the volume and the surface density. The masses of the parts of the disk and the whole which are interior to the equipotential $\xi=\xi_{0}$ are [8]:

$$
M_{\mathrm{disk}}\left(\xi_{0}\right)=\frac{a}{G}\left[\Phi_{\mathrm{K}}\left(\xi_{0}\right)-\Phi_{\mathrm{K}}(a)\right], \quad M_{\mathrm{total}}\left(\xi_{0}\right)=\frac{\xi_{0}}{G}\left(\xi_{0}-a\right) \Phi_{\mathrm{K}}^{\prime}\left(\xi_{0}\right) .
$$

Generally the disk mass becomes a progressively smaller part of the whole at large distances as $\xi_{0}$ increases. For example, if we choose $\Phi_{\mathrm{K}}(\xi)$ to be a logarithmic potential so as to obtain a rotation curve which becomes flat at large distances, then

$$
\Phi_{\mathrm{K}}(\xi)=V_{0}^{2} \ln \xi, \quad \rho=\frac{V_{0}^{2}}{4 \pi G \xi^{2}}+\frac{a V_{0}^{2} \delta(z)}{2 \pi G\left(R^{2}+a^{2}\right)} .
$$


Both disk and halo masses are now infinite, though the disk mass grows logarithmically with increasing $\xi_{0}$, while the total mass has a faster linear growth. An intermediate case between this, and that of Kuzmin's disk for which all of the mass is in the disk, is given by

$$
\Phi_{\mathrm{K}}(\xi)=-\frac{G M_{D}}{\sqrt{a \xi}} .
$$

Now the disk has finite total mass $M_{D}$, while the halo has infinite mass.

\subsection{Dynamics in Kuzmin-like Potentials}

Motion in a spherical potential is super-integrable. It conserves the energy $E$ and the angular momentum vector about the center of force. The motion is confined to the plane through the center of force and perpendicular to the angular momentum vector. Disk-crossing orbits in a Kuzmin-like potential pass continually back and forth between the regions $z>0$ and $z<0$, and hence from one spherical potential field to the other. The angular momenta which are conserved in the two regions are both described by the vector

$$
\mathbf{J}=[\mathbf{r}+a \operatorname{sgn}(z) \mathbf{k}] \times \mathbf{v}
$$

where $\mathbf{r}$ is the position relative to the origin, $\mathbf{v}$ is velocity, and $\mathbf{k}$ is the unit vector in the $z$-direction. This vector changes discontinuously as $z$ changes sign unless $\mathbf{v}$ is then perpendicular to the disk. The only component of $\mathbf{J}$ which does not change as the disk is crossed is the $z$-component $J_{z}$. That is because it is also the $z$-component $L_{z}$ of angular momentum $\mathbf{L}=\mathbf{r} \times \mathbf{v}$ about the origin midway between the two centers of force, and is a constant of the motion everywhere, as in any axisymmetric potential. Since it is known that energy and angular momenta are the only constants of motion in general spherical potentials and the other two components of $\mathbf{J}$ are not conserved in Kuzmin-like potentials, it follows that, contrary to what is stated in [9], Kuzmin-like potentials are not generally integrable. Kuzmin's disk is exceptional. It is integrable because its potential is Stäckel, and so has a third integral. That third integral is a linear combination of $\mathbf{J}^{2}$ and the $z$-component of the Laplace-Runge-Lenz vector, the fifth integral of a Keplerian potential [18].

\subsection{Orbits in Kuzmin-like Potentials}

Orbits must be computed numerically. We use the method described in [8] to compute surfaces of section (SOS) by integrating orbits from one crossing to the next, with no interpolation needed to find when and where crossings occur. The SOS show periodic orbits, regular regions associated with stable periodic orbits, and stochastic regions. One well-known family of periodic orbits which appears in many surfaces of section, and whose dynamics are particularly simple in a Kuzmin-like potential, is that associated with the period 1 periodic orbit of banana/saucer type [19]. We label a periodic orbit by the number of times that it crosses $z=0$ with $\dot{z}>0$ during a complete period of its motion in both $R$ and $z$. The banana periodic orbit, shown in the left panel of FIGURE 1, is a polar orbit with zero component $L_{z}$ of angular momentum about the axis of symmetry. It moves in a straight line directly towards or away from the center of force while on one side of the disk. Its two linear segments 

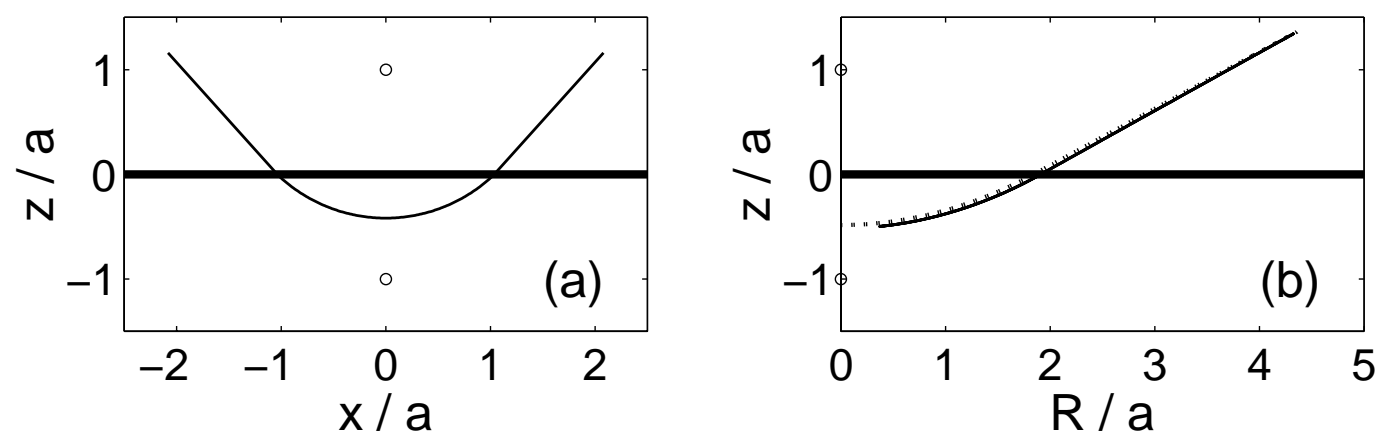

Figure 1: (a) A banana periodic polar orbit for Kuzmin's disk, with two exactly linear segments. This orbit is possible only if $E>-G M / 2 a \sqrt{2}$. It is drawn for the slightly larger value $E=-G M / 3 a$ when the curved segment is almost circular. The open circles mark the centers of force. (b) The full curve shows the saucer orbit for the logarithmic Kuzmin-like potential (6) for $R_{c}=3 a$ and $L_{z} / L_{c}(E)=0.2$. For comparison, the dotted curve shows half of the $L_{z}=0$ polar orbit for the same potential and $R_{c}$.

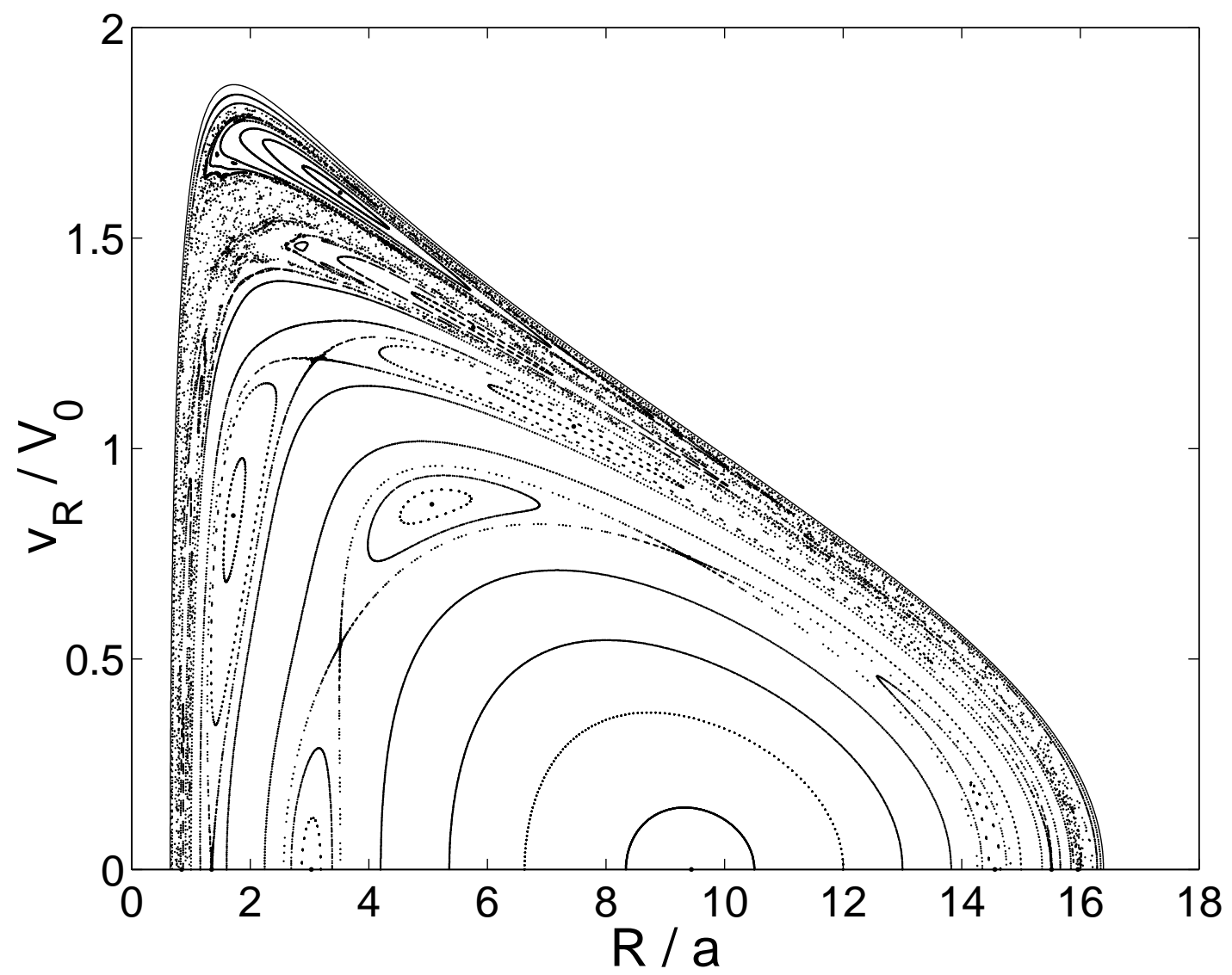

Figure 2: Surface of section for the logarithmic Kuzmin-like potential (6) for $R_{c} / a=10$ and $L_{z} / L_{c}(E)=0.15$. 
are connected by a curved segment on the other side of the disk when it is controlled by the other center of force. This banana orbit is modified to a saucer orbit when $L_{z} \neq 0$. Angular momentum then bars the orbit from approaching the axis of symmetry, and reflects it before it reaches the $z$-axis [19]. The right half of FIGURE 1 shows how little the orbit is changed otherwise; its abrupt change in curvature at the disk crossing is still evident, and its upper segment is close to straight.

FiguRE 2 displays the $z=0$ SOS for orbits in the logarithmic Kuzmin-like potential (6) for a specific pair of values of the energy $E$ and $L_{z}$. We identify the energy by the value of $R_{c}$, the radius of a circular orbit of that energy. This gives a typical distance of the orbit from the center of the model, and is more easily comprehensible than a numerical value of $E$. The value of $E$ can be computed from it using

$$
E=\Phi_{\mathrm{K}}\left(\xi_{c}\right)+\frac{R_{c}^{2}}{2 \xi_{c}} \Phi_{\mathrm{K}}^{\prime}\left(\xi_{c}\right), \quad \xi_{c}=\sqrt{R_{c}^{2}+a^{2}}
$$

We express $L_{z}$ as a fraction of the angular momentum

$$
L_{c}(E)=R_{c}^{2} \sqrt{\frac{\Phi_{\mathrm{K}}^{\prime}\left(\xi_{c}\right)}{2 \xi_{c}}},
$$

of the circular orbit of radius $R_{c}$, i.e. as a fraction of its maximum possible value.

Low values of $L_{z} / L_{c}(E)$, such as we have in our SOS, allow orbits which range widely, and have a range of geometrical shapes. They include orbits which are flat and remain close to the plane of the disk at all times. In fact the outer boundary of each SOS is formed by the orbit that lies always in the disk; a low value of $L_{z} / L_{c}(E)$ allows its pericenter to be a small fraction of $R_{c}$. Generally orbits become progressively flatter from the center point of an SOS to its outside. The center point shows the single crossing of a symmetrical period 1 thin tube orbit. This orbit travels far above and below the plane of the disk. It is closest to the $z$-axis at its extremities, and furthest when crossing the disk. We plot only the upper $v_{R} \geq 0$ half of each SOS, so as to show more detail; the lower $v_{R}<0$ half is its mirror image reflected in the $R$-axis.

The disk contains $25 \%$ of the matter inside the equipotential $\xi=\xi_{c}$ when $R_{c} / a=10$, and the angular momentum for FIgURE 2 is $15 \%$ of its maximum. The figure shows a large regular region of short axis tubes surrounding the thin tube orbit represented by the central fixed point at $R / a=9.44, v_{R}=0$. That orbit rises to distances $z / a= \pm 9.76$ above and below the disk, at which points $R / a=1.64$. It remains always far from the center. Two four-island chains in the regular region surround pairs of period 2 orbits. Those for the inner chain are of the symmetric spaceship type shown by full curves in FiguRE 7(b) of [8]. The reason for four islands, but period 2, is that the orbits can be traversed in either direction. Those for the outer chain, for which the stability properties are reversed, are a pair of unsymmetric twisted fish form, shown by dashed curves in FiguRE 7(b) of [8]. Orbits cross the disk closer and closer to its center as we move outwards through the SOS, as well as remaining progressively closer to the disk. They eventually become primarily chaotic when they approach closer to the $z$-axis than $R / a \approx 1$. The two families of regular orbits within the chaotic sea are a three-island chain associated with period 3 pretzel orbits [19] at $R / a=5.76, v_{R} / V_{0}=1.29$, and the outer two-island chain associated with the period 1 
saucer orbit at $R / a=3.52, v_{R} / V_{0}=1.61$. The latter is flatter than that shown in FigURE 2(b). Adjacent to outer boundary of the SOS is a narrow strip of regular orbits which remain very close to the plane of the disk at all times.

FIGURE 11 of [8] displays the SOS for the same potential and energy but the lower angular momentum $L_{z} / L_{c}(E)=0.1$. It is not greatly different, but its chaotic region is larger and now envelops the outer four-island chain. The extent of the chaotic region shrinks as $L_{z} / L_{c}(E)$ increases, and the four-island chains have disappeared before $L_{z} / L_{c}(E)=0.2$. The reason for their disappearance is that they merge and annihilate at $L_{z} / L_{c}(E)=0.184$ in the manner discussed in Terzić [20]. That same merger is close to happening in FiguRE 11 below for a model of the Milky Way.

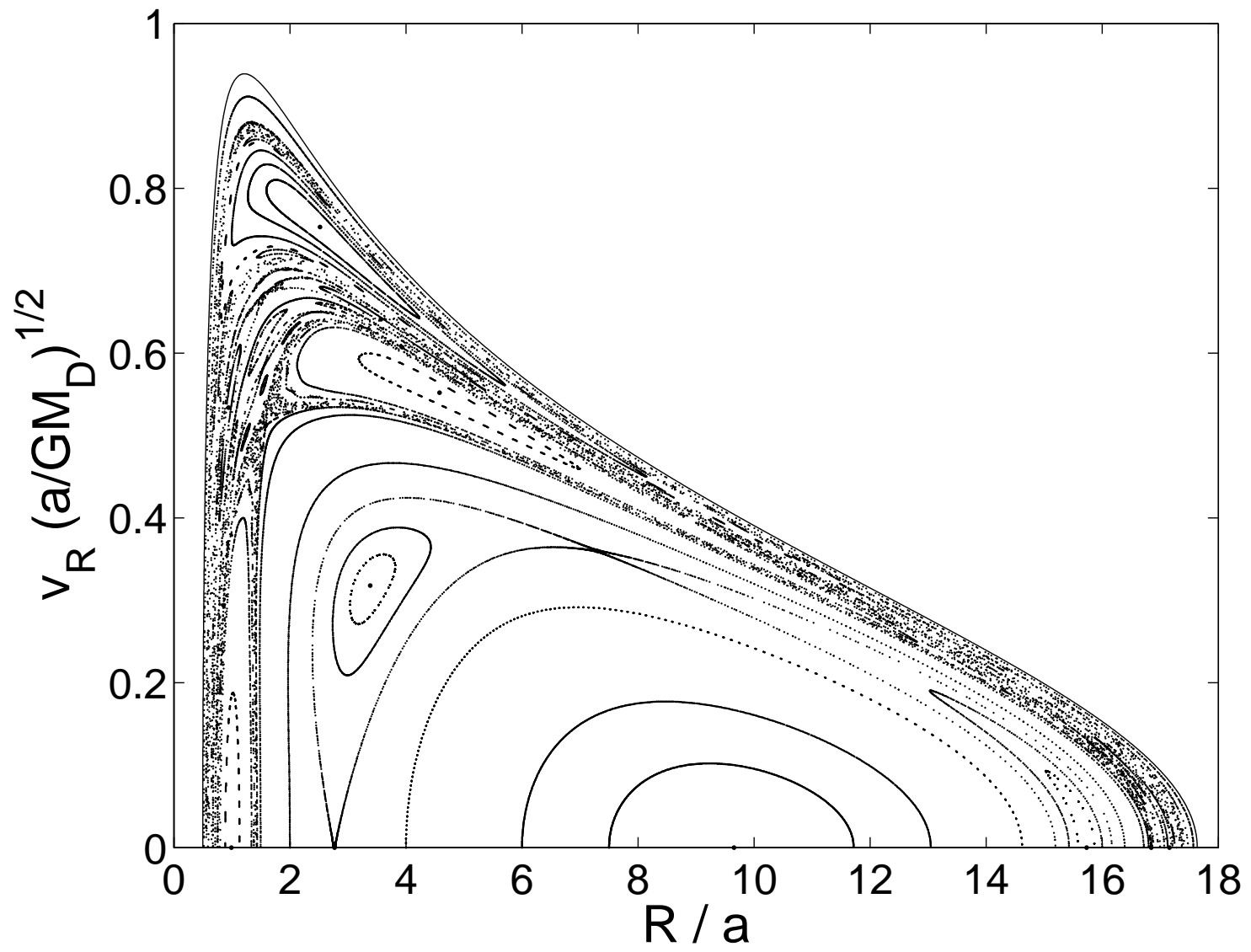

Figure 3: Surface of section for the power-law Kuzmin-like potential (7) for $R_{c} / a=10$ and $L_{z} / L_{c}(E)=0.15$.

The extent of the chaotic region decreases at lower energies, but it is at least equally important in Figure 3 for the power law potential (7) as it is in Figure 2. Figure 3 is drawn for the same $R_{c}$ and relative angular momentum as FigURE 2, though now the disk has only $18 \%$ of the matter inside $\xi=\xi_{c}$. It lacks the period 2 orbits of Figure 2 , but has a three-island chain associated with period 3 reflected fish orbits [19] in its regular region. The chaotic region surrounds prominent islands of stability associated with the saucer orbits and a second three-island periodic chain, as well as many smaller higher order resonances. 
The stable orbit at the center of the outer three-island chain is again of pretzel type, like the unstable period 3 orbit of the inner chain. The two three-island chains will also merge and annihilate at higher angular momenta as in Terzić [20].

\section{OTHER SIMPLE MODELS}

\subsection{Miyamoto-Nagai models}

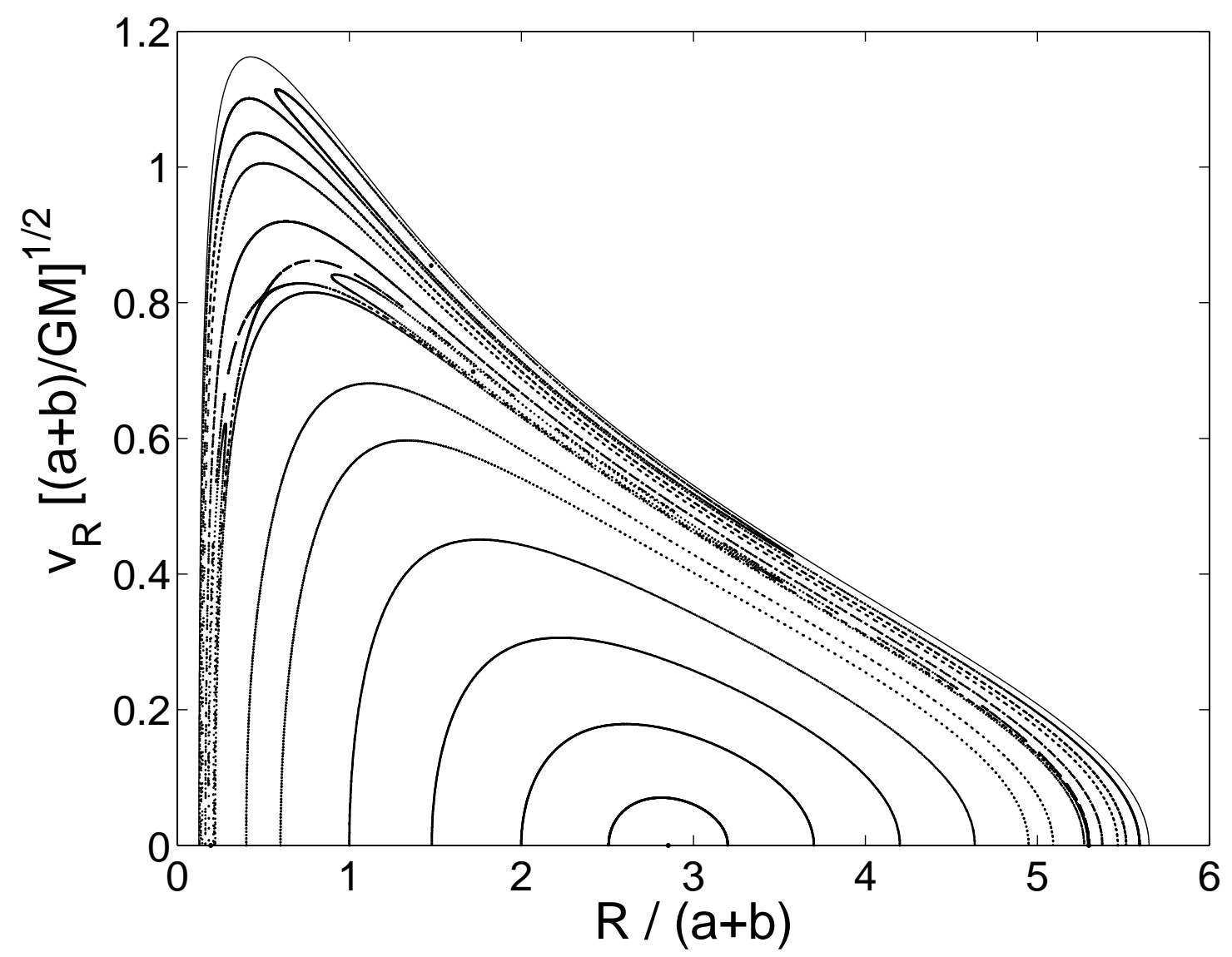

Figure 4: Surface of section for the Miyamoto \& Nagai potential (11) for $R_{c} /(a+b)=3$ and $L_{z} / L_{c}(E)=0.1$.

Miyamoto \& Nagai proposed the potential

$$
\Phi_{\mathrm{MN}}=-\frac{G M}{\xi_{b}}, \quad \xi_{b}=\sqrt{R^{2}+\left(a+\sqrt{z^{2}+b^{2}}\right)^{2}},
$$

with two length scales $a$ and $b$ for modelling galaxies. It has been used widely. Binney \& Tremaine [17] comment that the flattened isophotes obtained for $b / a=0.2$ are qualitatively similar to the light distributions of disk galaxies. Johnson, Spergel \& Hernquist [13] use a Miyamoto-Nagai potential with $b / a=0.04$ in their model of our Galaxy, while Patsis et al. [14] use one with $b / a=1 / 3$ for their explanation of boxy profiles in normal spirals. 
The Miyamoto-Nagai potential links Kuzmin's disk, its $b=0$ case, to Plummer's sphere [21], which is its $a=0$ case. Both of these limiting cases are integrable, although their third integrals differ. We have looked for, but failed to find, evidence of chaos at intermediate cases. FiguRE 4 is typical. It is for $a=b$, which is halfway between the two integrable extremes, and for a low relative angular momentum to allow a wide range of orbits. The SOS is dominated by thin tubes, interrupted only by two slender island chains around the period 1 saucer and period 3 pretzel orbits.

Alar Toomre (private communication) proposed a combination of a Kuzmin disk of mass $\delta M$ and a Plummer sphere of mass $(1-\delta) M$ with the same length scale as an alternative link between Kuzmin's disk and Plummer's sphere. We computed some of its SOS for different $\delta$, which we do not show because we found them also to be highly regular.

\subsection{Thickened Kuzmin-like Potentials}

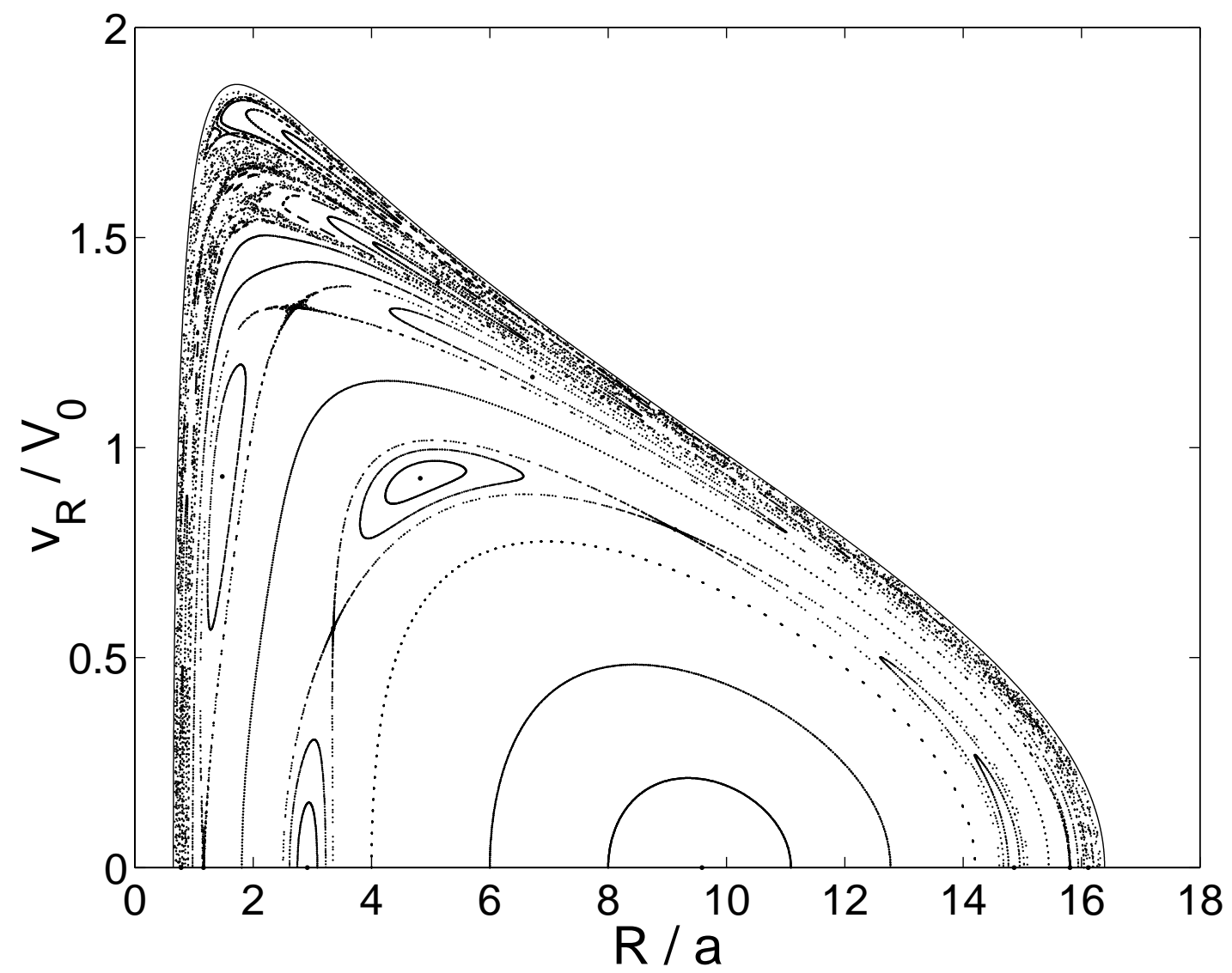

Figure 5: Surface of section for the thickened logarithmic Kuzmin-like potential (6) for $R_{c} /(a+b)=10, L_{z} / L_{c}(E)=0.2$, and $b / a=1 / 3$.

Non-zero values of the length scale $b$ in the Miyamoto-Nagai variable $\xi_{b}$ of equation (11) thicken Kuzmin's disk. The Kuzmin-like potentials of $\S 2.2$ can similarly be thickened by 
replacing $\xi$ in the Kuzmin-like potential equation (2) by $\xi_{b}$ to get

$$
\Phi=\Phi_{\mathrm{K}}\left(\xi_{b}\right) .
$$

FiguRE 5 shows the SOS that is obtained when the logarithmic Kuzmin-like potential with the same $E$ and $L_{z}$ as in FiguRE 2 is thickened with $b / a=1 / 3$. Overlapping the two figures shows that the quite substantial thickening has enlarged the regular central region only a little at the expense of the chaotic region, the islands within the chaotic sea are somewhat diminished, but the basic qualitative structure is unchanged. FIGURE 5 provides further evidence, in addition to that in [8], that the chaos which we found to be induced by disk crossings is not due to the use of a razor-thin disk, because it persists when the disk is thickened significantly.

\subsection{Scalefree Logarithmic models}

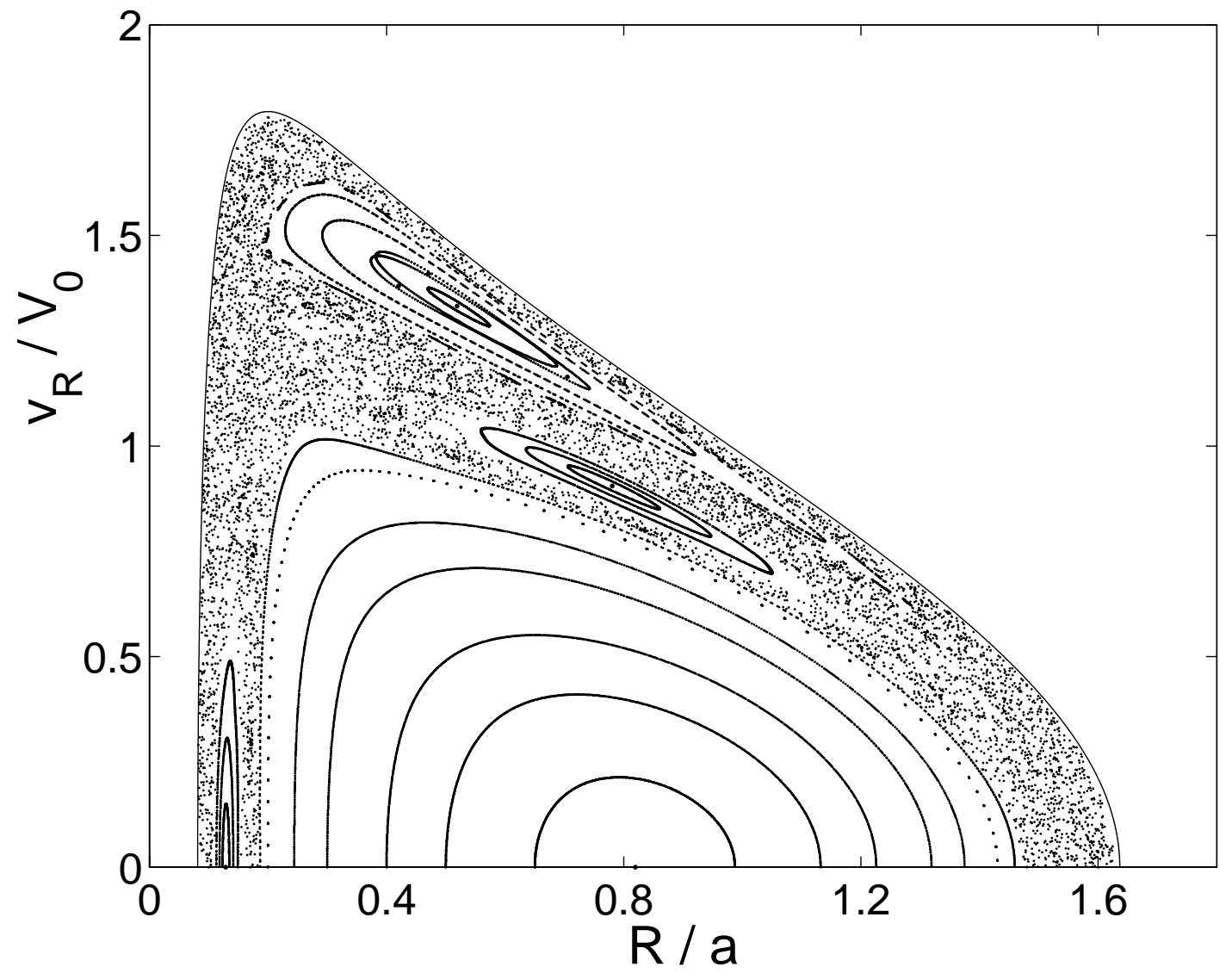

Figure 6: Surface of section for the $n=0$ scalefree logarithmic potential (13) for a $20 \%$ disk $(\beta=0.2)$, and $L_{z} / L_{c}(E)=0.2$.

The last class of simple models which we consider are the scalefree logarithmic models of Monet et al. [11] and Toomre [12]. They have potentials and densities of the form

$$
\Phi=V_{0}^{2}[\ln (r / a)+P(\theta)], \quad \rho=\rho_{0} S(\theta)\left(\frac{a}{r}\right)^{2},
$$



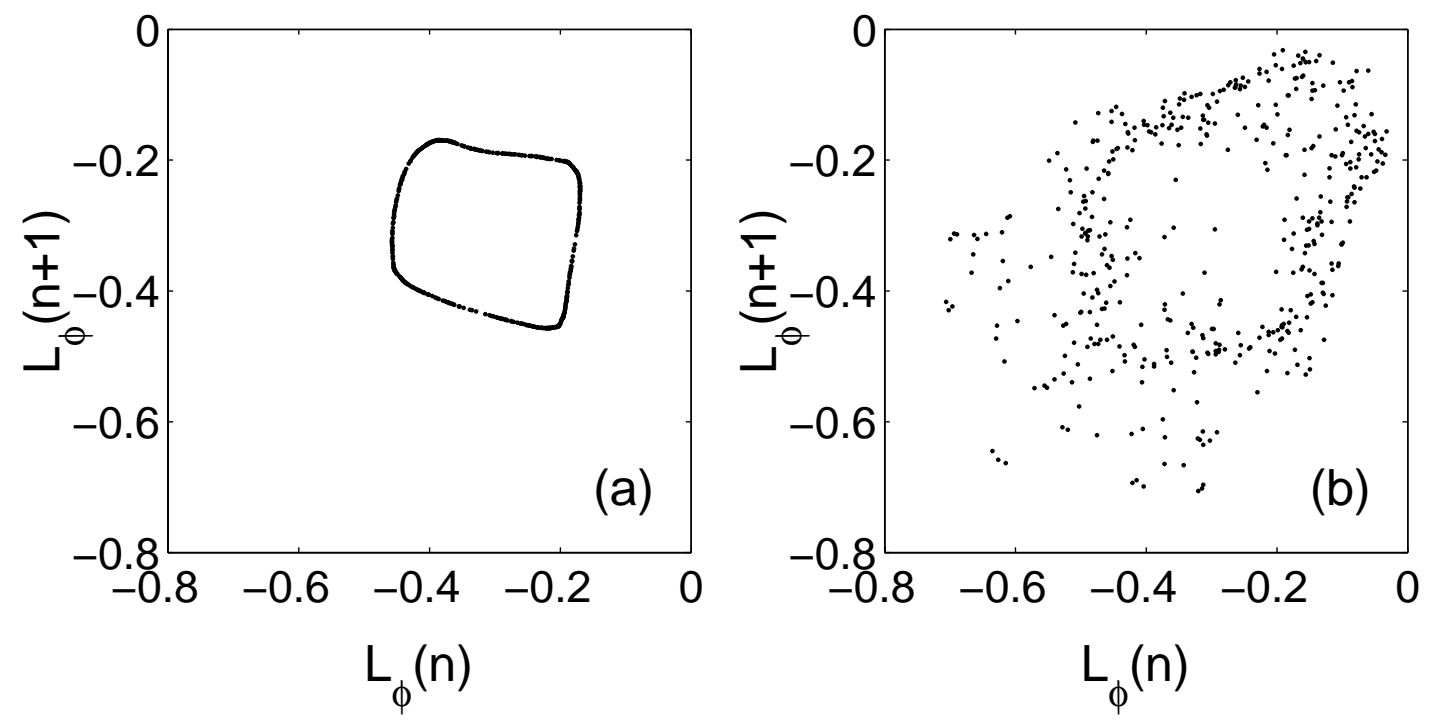

Figure 7: Delay plots of $L_{\phi}$, the angular momentum perpendicular to the axis of symmetry, when the SOS is crossed with $\dot{z}>0$. The two orbits start in the SOS of Figure 6 from (a) $R / a=0.4, v_{R} / V_{0}=1.314$, and (b) $R / a=0.4, v_{R} / V_{0}=1.312$.

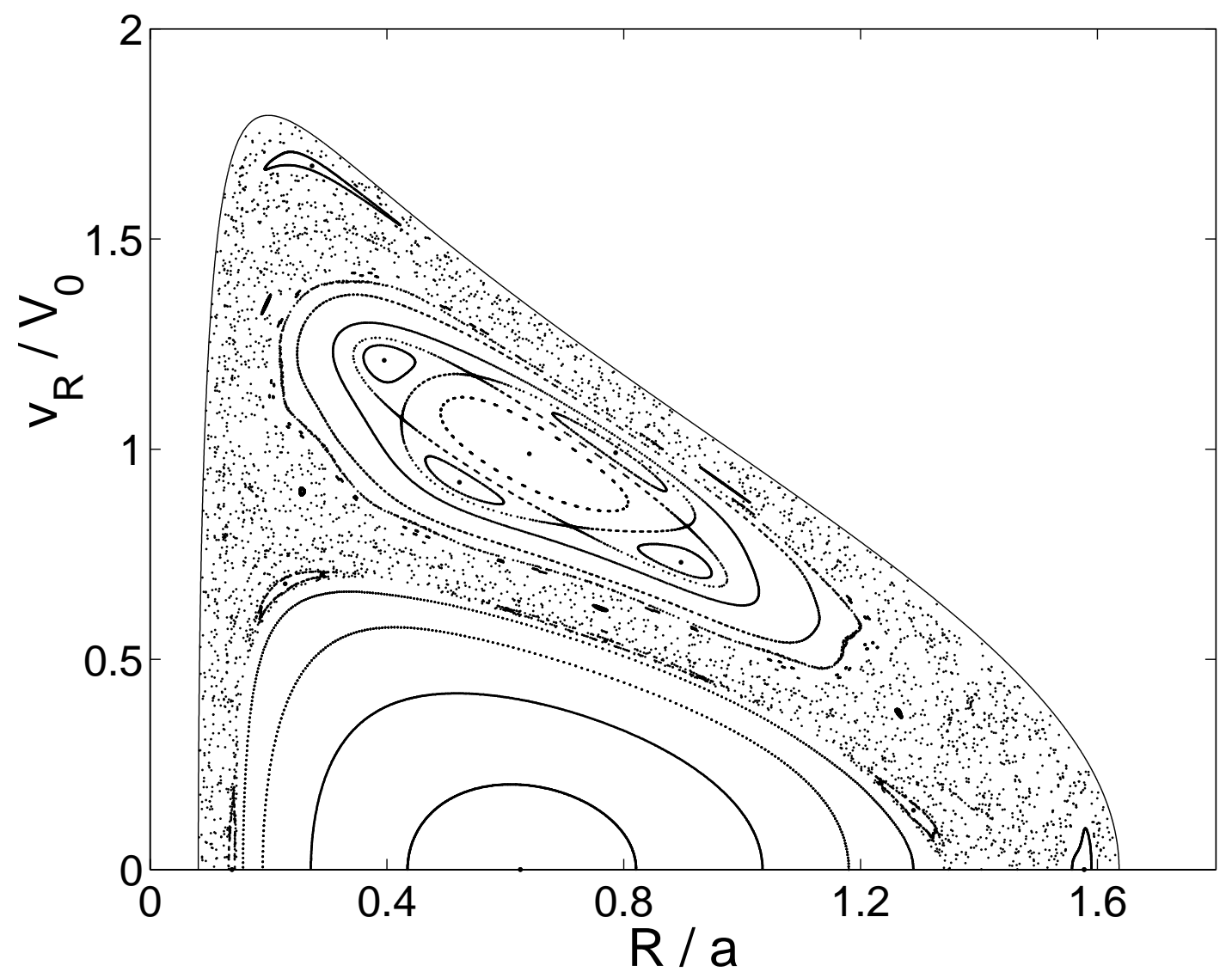

Figure 8: Surface of section for the $n=1$ scalefree logarithmic potential (13) for a $20 \%$ disk $(\beta=0.2)$, and $L_{z} / L_{c}(E)=0.2$. 


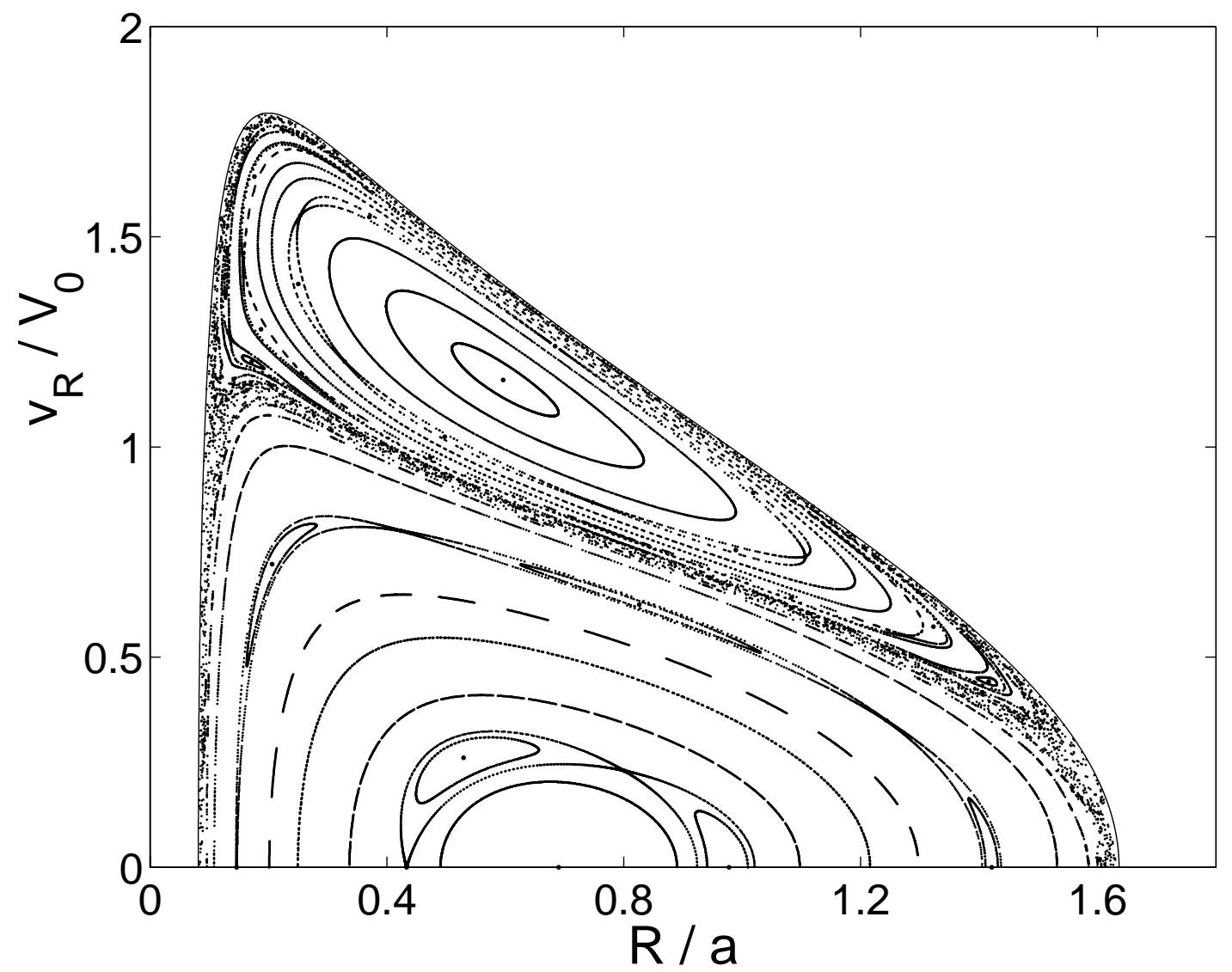

Figure 9: Surface of section for the $n=1$ scalefree logarithmic potential (13) with no disk $(\beta=0)$ and $L_{z} / L_{c}(E)=0.2$. 
where $\theta$ is the colatitude measured from the axis of symmetry, and $\rho_{0}$ is the average density on the sphere $r=a$. These models can incorporate a razor-thin disk in the equatorial plane by including a singular component $2 \beta \delta(\theta-\pi / 2)$ in the angular density function $S(\theta)$. The disk then contains a fraction $\beta$ of the mass within any finite radius because of the scalefree property. The total mass of both disk and halo are now infinite.

Toomre's models are more general and are constructed so as to have two-integral distribution functions

$$
f\left(E, L_{z}\right) \propto L_{z}^{2 n} \exp \left[-\frac{(2 n+2) E}{V_{0}^{2}}\right] .
$$

Monet et al. consider only the $n=0$ case, for which the distribution function depends only on the energy because, as Toomre notes, the density is then constant on the equipotentials. The volume densities (3) of Kuzmin-like models are also constant on equipotentials, and so their two-integral distribution functions, which we do not give, also depend only on energy. The $n=0$ scalefree models are not Kuzmin-like. Monet et al. introduced their models to investigate the effect of massive disks on bulge isophotes. The mass models (13) become flatter and the density increasingly concentrated towards the equatorial plane with increasing $n$. Toomre's Figure 1 shows this for the diskless $\beta=0$ case, and the same happens when there is a disk.

Neither Monet et al. nor Toomre discuss orbits in their models. Scalefree models have the advantage that orbits at each energy are scaled versions of those at other energies [22]. SOS are therefore needed for just one value of $E$. We take that value to be $V_{0}^{2} / 2$, corresponding to $R_{c} / a=1$. That still leaves three parameters, the angular momentum $L_{z}$, the relative mass $\beta$ of the disk, and the index $n$, to be explored. Our three SOS, all with the same $L_{z} / L_{c}(E)=0.2$, study the effects of varying halo flattening and disk mass. FigurE 6 is for the otherwise spherical $n=0$ halo flattened by a $20 \%$ disk. Its SOS is simpler than that for a logarithmic Kuzmin-like potential, though it too is for a logarithmic potential. The regular and chaotic regions are now equally large. The chaotic region is interrupted only by a prominent family of saucer orbits, and a smaller family of period 3 pretzel orbits. The slender three-island chain surrounding the saucer orbit is associated with a periodic orbit which has a small period 3 oscillation about the saucer. Increasing the disk mass enlarges the chaotic region, while decreasing the disk diminishes the chaotic region. Chaos of course disappears when there is no disk and the halo becomes spherical.

FiguRE 7 shows delay plots for two orbits which lie on the lower edge of the saucer orbit region of FIGURE 6 . Delay plots are used widely for analyzing time series generated by dynamical systems [6], and can show more clearly than trajectories in $(R, z)$-space which orbits are chaotic. FIGURE 7 plots the azimuthal component of angular momentum $L_{\phi}=$ $-R \dot{z}$ at one crossing of the SOS with $\dot{z}>0$, versus its value at its previous crossing. The role of $L_{\phi}$ here is simply that of a scalar property of the orbit. It varies continuously along the orbit, unlike the $\mathbf{J}$ components used in the delay plots in [8] which remain constant in $z>0$ and change only because of disk crossings. Nevertheless both kinds of delay plots have the same character. Those for regular orbits form closed loops, while those for chaotic orbits show a widespread scatter. FiguRE 7 shows how sudden the transition can be; part (a) shows some breaking up from the closed loop characteristic of a saucer orbit, while (b) for a close starting point in the SOS shows nearly random scattering with little evidence of the previous structure. 
FIGURE 8 shows the SOS for the flatter $n=1$ halo, also with a $20 \%$ disk. The regular core has shrunk, and the general extent of the chaotic region has grown, but it now embeds a much larger regular island. That island has the usual saucer orbit at its center, and it is now surrounded by islands of period 4 orbits which wobble around the saucer orbit. The pretzel family seen in FiguRE 6 has disappeared, but other small islands have appeared, including a fish orbit at $R / a=0.27, v_{R} / V_{0}=1.67$.

Figure 9 , also for $n=1$ but now with no disk, shows the extent to which the features seen in FigurE 8 are due to halo flattening alone. It shows that the disk is responsible for the large size of the chaotic region. With no disk, chaos is confined to narrow strips around the regular regions, and the neighborhoods of some unstable fixed perodic orbits. The absence of the disk has allowed a large variety of periodic orbits and island chains to appear, including a period 3 reflected fish orbit near the center, and two four-island chains now surround the saucer orbit.

\section{MODELS OF THE GALAXY}

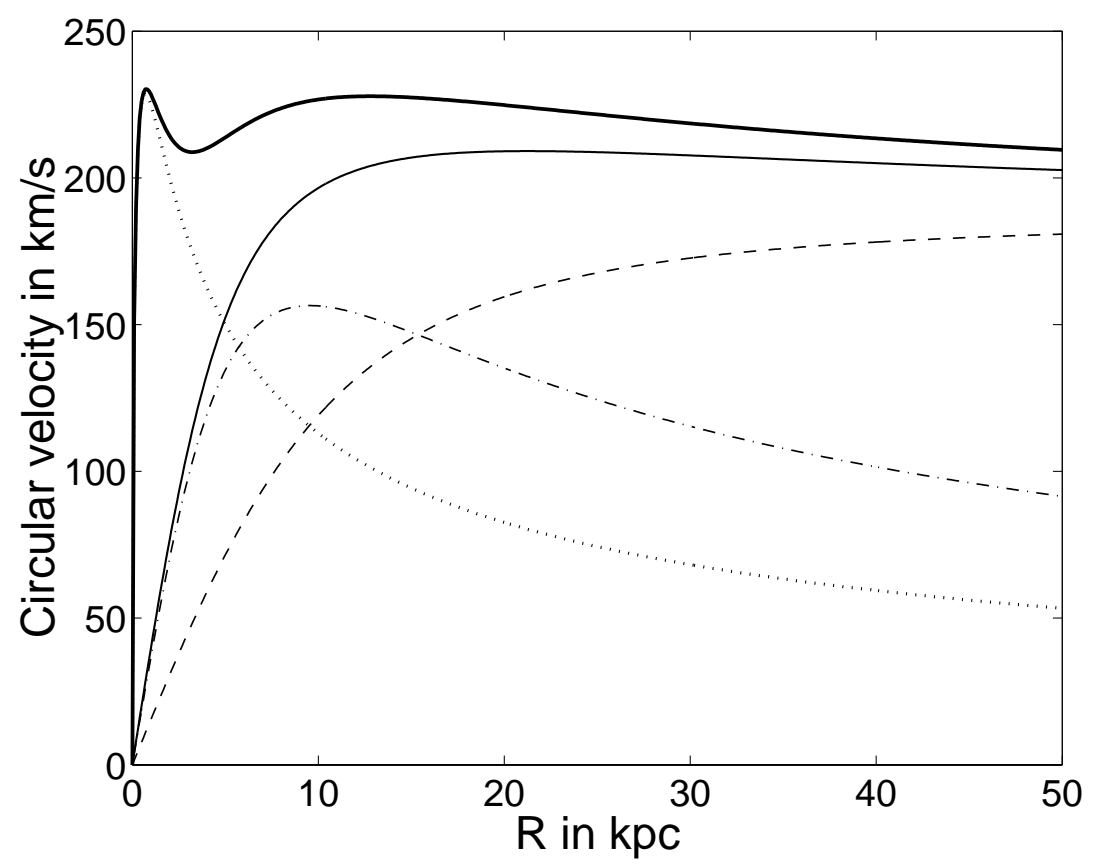

Figure 10: Rotational velocities of the components of models of the Galaxy. The dot-dashed, dashed, and dotted curves are those due to the disk, halo, and bulge respectively. The thin full curve is that for the disk plus halo model used in [15]. The thick full curve is that for the full three-component model.

We investigate orbits in the three-component model of the Galaxy introduced by Johnston et al [13], and extended by Helmi [23], [24] to allow for halo flattening, with potential

$$
\Phi=\Phi_{\mathrm{MN}}-\frac{G M_{\text {bulge }}}{r+c}+V_{\text {halo }}^{2} \ln \left(d^{2}+R^{2}+\frac{z^{2}}{q^{2}}\right) .
$$




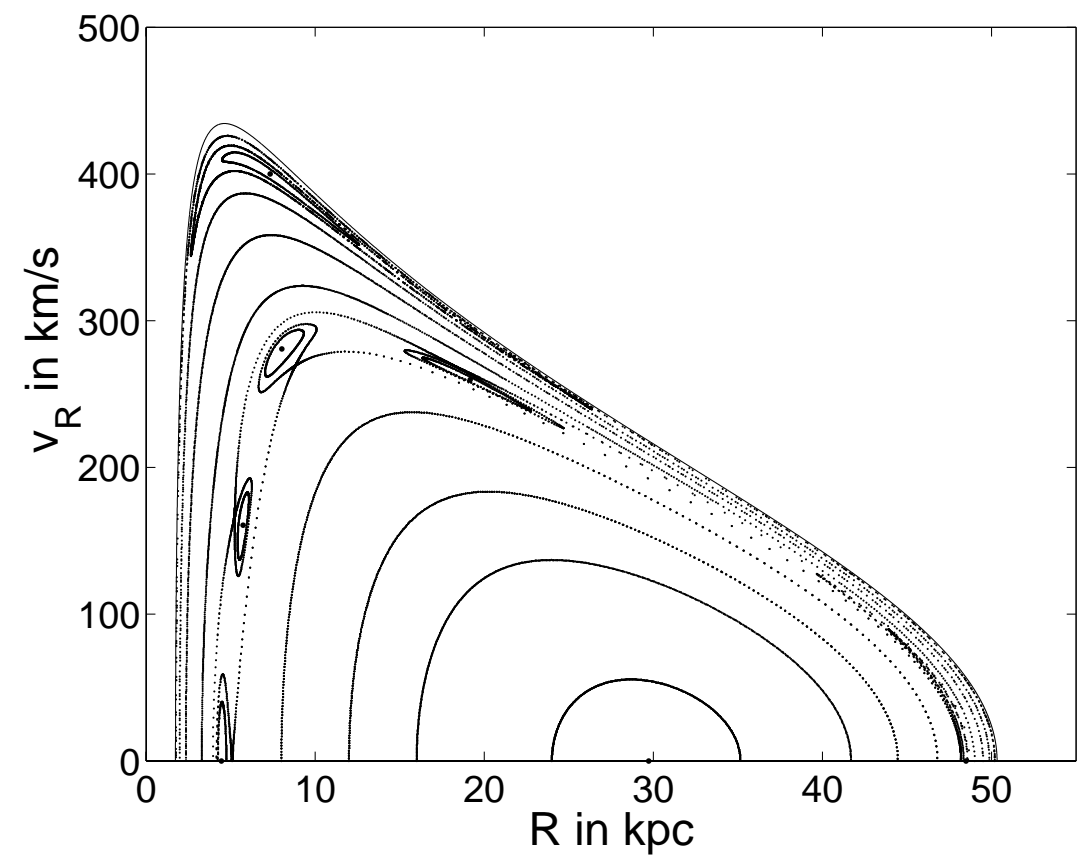

Figure 11: Surface of section for the Galactic potential (15) with a spherical halo $(q=1)$, $R_{c}=30 \mathrm{kpc}$ and $L_{z} / L_{c}(E)=0.15$.

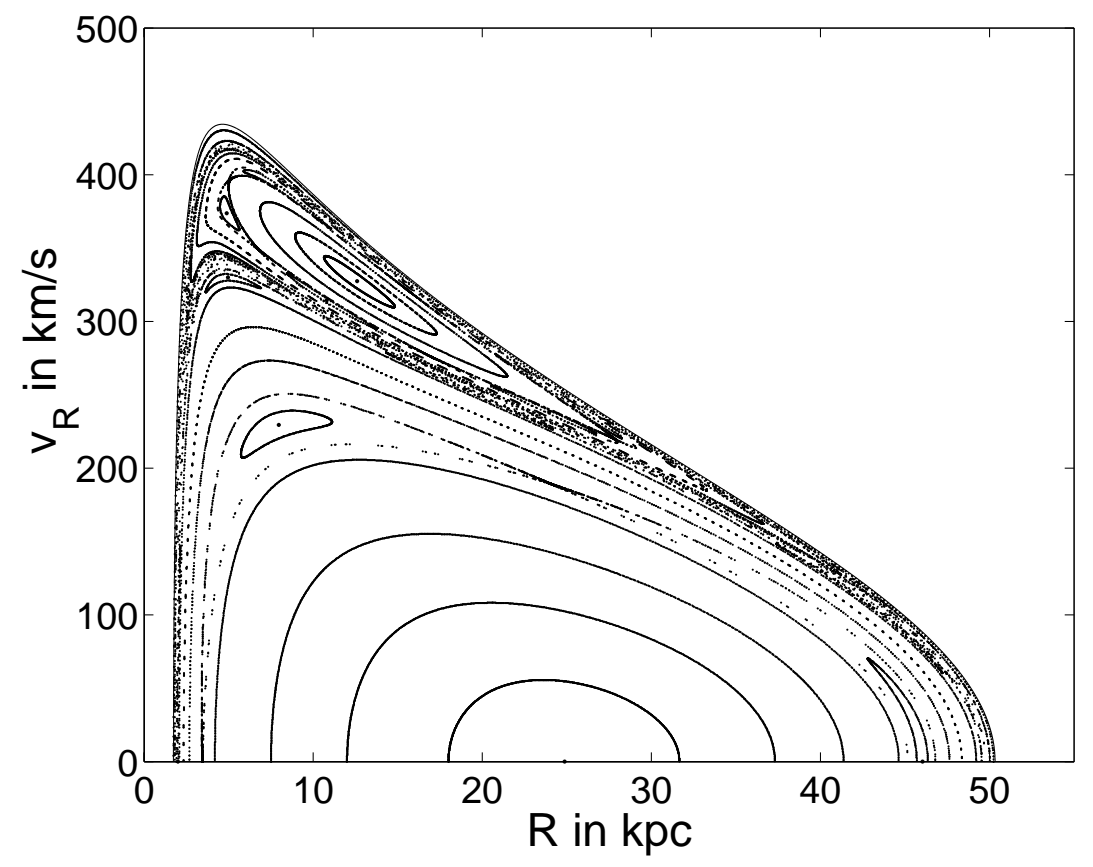

Figure 12: Same as Figure 11, but with an oblate spheroidal halo with $q=0.8$. 


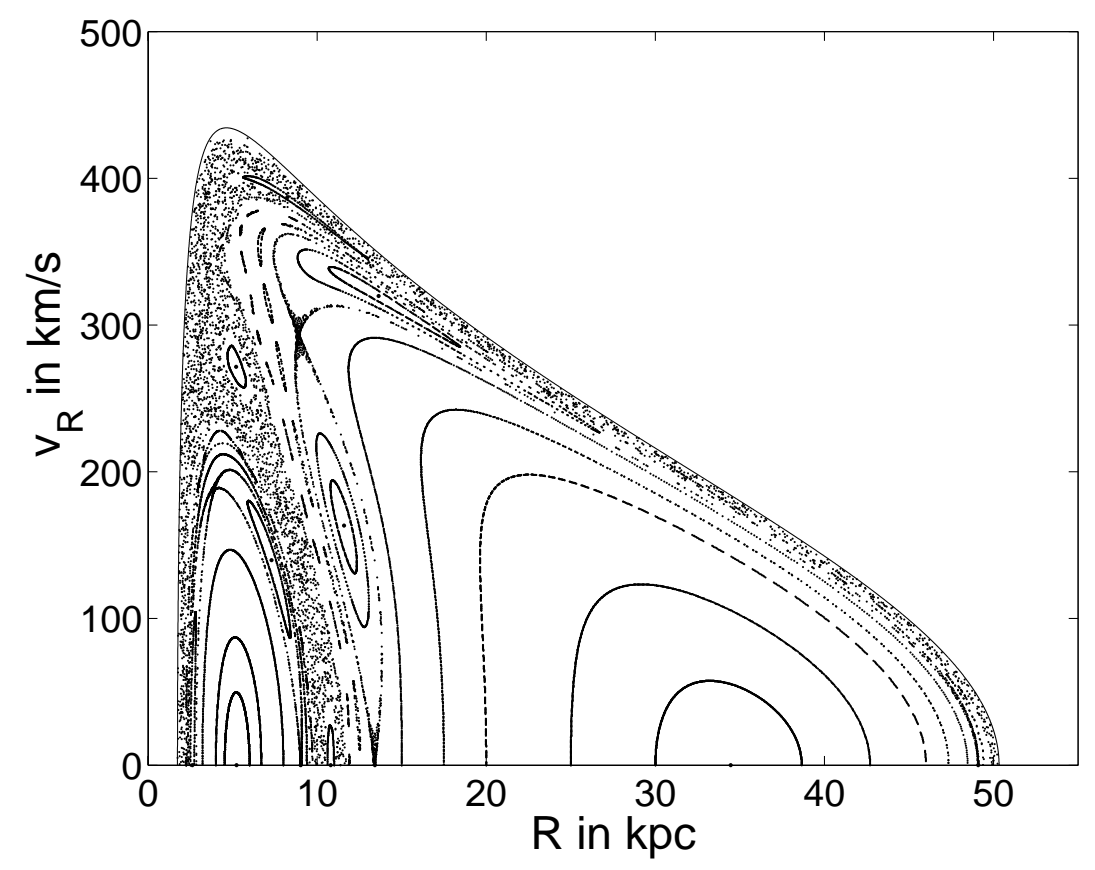

Figure 13: Same as FiguRE 11, but with a prolate spheroidal halo with $q=1.25$.

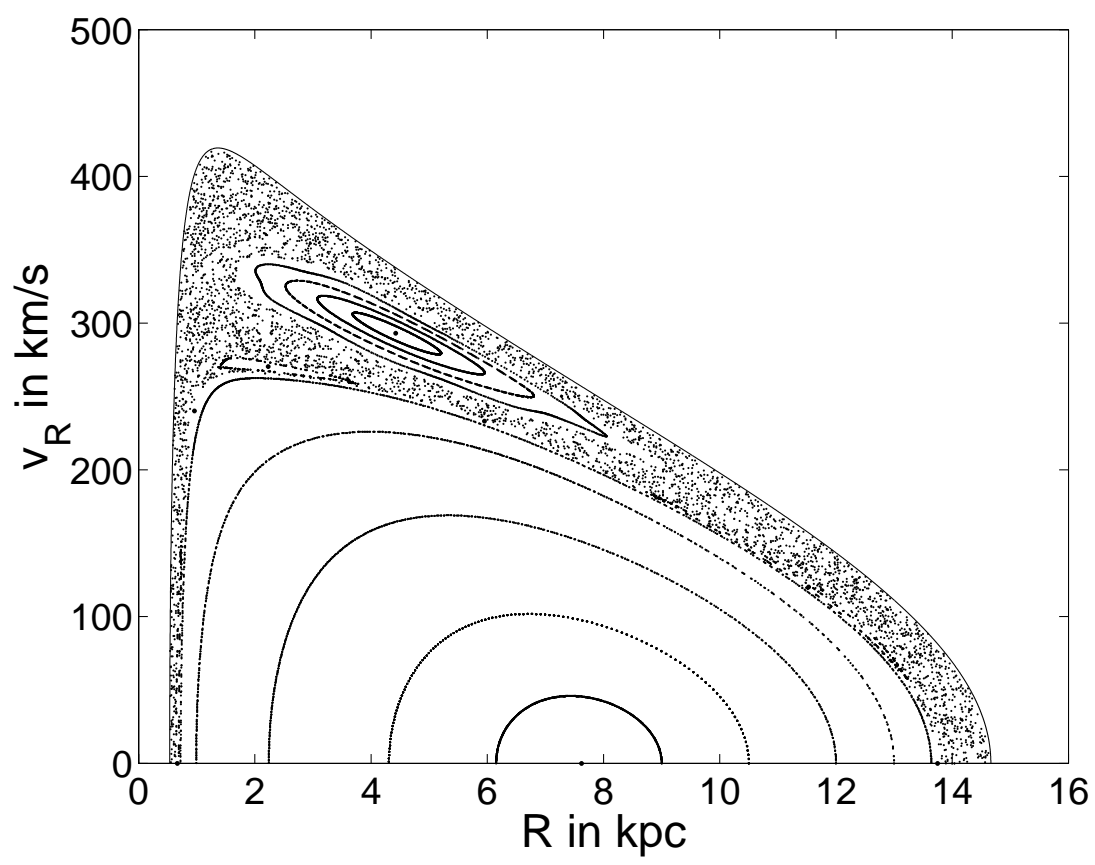

Figure 14: Same as Figure 11, but at the lower energy for which $R_{c}=9 \mathrm{kpc}$. 
The rotation curves obtained from its components and their combinations are shown in Figure 10. The mass of the bulge is 0.34 times that of the disk and has a length scale $c=0.7 \mathrm{kpc}$, versus $a+b=6.76 \mathrm{kpc}$ for the disk and $d=12 \mathrm{kpc}$ for the halo. The centrally concentrated bulge dominates the potential near the center, and thereby distinguishes the potential (15) from those we considered earlier.

The SOS in FiguRES 11 through 13 are all for an energy for which $R_{c}=30 \mathrm{kpc}$, and a relative angular momentum of $L_{z} / L_{c}(E)=0.15$ as earlier. The reason for that choice of $R_{c}$ is that it corresponds approximately to that of four of the six orbits studied in Helmi \& White [15], though they had only a disk and spherical halo, and no bulge. When we too ignored the bulge, we obtained a purely regular SOS with no prominent resonances. FigURE 11 shows the effect of adding the bulge. The SOS is still regular, but has three periodic orbits in addition to the ever-present thin tube; a saucer orbit near its edge, and a pair of period 2 orbits like those of FIgURE 2. The period 2 orbits are about to merge and disappear, as do those of Figure 2 at a higher $L_{z}$.

The consequences of making the halo spheroidal are illustrated in the next two figures. Figure 12 shows that oblateness enlarges the saucer family of orbits, and introduces more periodic orbits, including a central period 3 reflected fish orbit like those in FIGURES 3 and 9, but that there is not much chaos at $L_{z} / L_{c}(E)=0.15$. More chaos develops at lower angular momenta when resonances grow and overlap. FiguRE 13 shows that prolateness makes a much bigger difference. It introduces new periodic orbits, and replaces old ones. The left hand regular region is centered on a thin inner long-axis tube [18]. The three-island chain around it is associated with a period 3 orbit which wobbles around that inner tube and is of the same type as that shown in Figure 7(c) of Evans [25]. The orbits associated with the right hand regular region are similar to those found in oblate potentials, with a thin outer long axis tube at its center, and period 2 twisted fish further out. There are no longer any unsymmetric period 1 saucer orbits; they have been replaced by the symmetric period 1 inner tube which crosses the SOS at $R=5.23 \mathrm{kpc}$, but then travels out to $R=27 \mathrm{kpc}$, and to distances $z= \pm 48 \mathrm{kpc}$ above and below the Galactic plane. The chaotic region is no longer confined to orbits in the outer part of the SOS which remain close to the Galactic plane. The band of chaotic orbits which lie between the two regular islands range to distances of over $50 \mathrm{kpc}$ above and below the Galactic plane.

FIGURE 14 is for a much lower energy, of the order of that of near circular orbits in the solar neighborhood, though with much lower angular momenta than them. Its SOS, which is for a spherical halo, is the simplest of all. It has a large chaotic region which becomes larger at lower energies and hence lower $R_{c}$. FIGURE 14 is somewhat like FiguRE 6 for the scalefree disk, but it shows no evidence of a three-island chain. Some differences are to be expected because the spherical bulge dominates the halo for the central regions of FiguRE 14. The singular halo of the scalefree model of FIGURE 6 is equally important everywhere, and it is not spherical because the disk gives it oblateness.

\section{CONCLUSIONS}

We have studied orbital behavior in a variety of galactic models with disks and halos. Crossing the disk causes an abrupt change in acceleration, but not in the velocity of an orbit. Hence 
disk crossing is a milder perturbation than the impulses, and resulting velocity changes, which Wisdom [26] used to explain the Kirkwood gaps in the asteroid belt. Nevertheless we find that repeated disk crossings can allow some orbits to become chaotic, and that this effect is not sensitive to disk thickness. The orbits which become chaotic are ones whose pericenters and apocenters are far apart, and whose trajectories are confined relatively closely to the plane of the disk. Kuzmin's disk is exceptional in that every one of its disk-crossing orbits is regular. The Miyamoto-Nagai disks appear to inherit a near-integrability from Kuzmin's disk and Plummer's sphere which they connect. All our other examples, with the single exception of FigurE 11 for a Miyamoto-Nagai disk in a spherical potential, have both purely regular regions, and chaotic regions which contain islands of regularity. There are many more islands than our SOS show, because more, smaller, and higher period, islands can always be found, given sufficient persistence. However we believe that our SOS do not omit any islands of significant size.

We show SOS with low values of $L_{z} / L_{c}(E)$ because only then can orbits range widely in $R$ or travel far from the plane of the disk. Chaotic regions diminish as $L_{z} / L_{c}(E)$ grows and orbits range less widely. The scalefree models of $\S 3.3$ enable us to vary the mass of the disk relative to that of the halo, and the inter-related flattening of the halo. Increased flattening increases the number of noticeable resonances, while increasing the disk mass enhances their overlap and the generation of chaos. Our earlier study [8] of Kuzmin-like potentials, in which there was no freedom to adjust the relative mass of the disk, showed more chaos at higher energies.

In restricting our study to the orbits of test particles, our calculations are simpler than those of Helmi \& White [15] and Helmi [23], [24] who also modeled the orbiting satellite galaxy. Helmi \& White found that their satellites soon disrupted so that its stars behaved as test particles for most of their evolution, as do our simple orbits. Aguilar, Hut \& Ostriker [27] modeled orbiting globular clusters, and found that the clusters which are most vulnerable to destruction by bulge and disk shocking are those on highly radial orbits which come closer and faster to the central regions of the Galaxy. That is consistent with our finding of chaotic regions in the outer parts of our SOS.

Our investigations of orbits in the model (15) of the Milky Way are of very limited scope. When the halo is spherical like the bulge, then only the disk perturbs the integrability of the potential. Chaos can not then be expected to arise unless an orbit approaches closely enough for the perturbing effect of the disk to be large. The disk enhances resonances at the energy used in Figure 11, but no chaos is evident. The less energetic orbits in FiguRE 14 show considerable chaos. This is largely the result of the interaction of the bulge with the Miyamoto-Nagai disk because the halo is much less important closer in.

It is well known that spheroidal halos introduce resonances and periodic orbits [25], and hence more scope for a disk to generate chaos. Comparing Figures 12 and 13 shows how much more effective a prolate halo is than an oblate one for generating chaos. The orbits used by Helmi [23], [24] in her modeling of the Sagittarius dwarf have pericenters in the range 10.1 to $14.7 \mathrm{kpc}$, and apocenters in the range 59.2 to $52.3 \mathrm{kpc}$ as $q$ varies from 0.8 to 1.25 . These ranges require somewhat larger values of $R_{c}$ than the $R_{c}=30 \mathrm{kpc}$ we have used, but the large size of the central regular region in FIGURE 12 for an oblate $q=0.8$ halo leaves little doubt that an orbit with $R_{\text {peri }}>10 \mathrm{kpc}$ will lie safely inside it and be regular. Although the chaotic region of the prolate $q=1.25$ halo in Figure 13 extends 
as far out as $R=11.7 \mathrm{kpc}$, the wide ranging orbits which pass through there have much smaller pericentric distances because they come closer than $3 \mathrm{kpc}$ of the center of the Galaxy elsewhere in their orbits. Hence orbits similar to those considered by Helmi are unlikely to chaotic even if the Galactic halo is prolate. Of course, the fact that the debris from the Sagittarius remnant is identifiable is direct evidence that its orbit is regular and not chaotic.

Acknowledgments This work was supported by the National Science Foundation grant DMS-0104751.

\section{References}

[1] Hubble, E.P. 1936. The Realm of the Nebulae. Yale University Press, New Haven, CT.

[2] Bender, R. 1988. Velocity anisotropies and isophote shapes in elliptical galaxies. Astron. \& Astrophys. 193: L7-10.

[3] Nieto, J.-L., M. Capaccioli \& E.V. Held. 1988. More isotropic oblate rotators in elliptical galaxies. Astron. \& Astrophys. 195: L1-4.

[4] Scorza, C. \& R. Bender. 1995. The internal structure of disky elliptical galaxies. Astron. \& Astrophys. 293: 20-43.

[5] Ostriker, J.P., L. Spitzer \& R.A. Chevalier. 1972. On the evolution of globular clusters. Astrophys.J. 176: L51-56.

[6] Alligood, K.T., T.D. Sauer \& J.A. Yorke. 1996. Chaos: an Introduction to Dynamical Systems. Springer. New York, NY.

[7] Martinet, L. \& F. Mayer. 1975. Galactic orbits and integrals of motion for stars of old Galactic populations. Astron. \& Astrophys. 44: 45-57.

[8] Hunter, C. 2003. Disk-crossing orbits. In Galaxies and Chaos, Lecture Notes on Physics, 626. G. Contopoulos \& N. Voglis, Eds.: 137-153, Springer. New York, NY.

[9] Tohline, J.E. \& K. Voyages. 2001. Integrals of motion in Kuzmin-like potentials. Astrophys. J. 555: 524-531.

[10] Miyamoto, M. \& R. Nagai. 1975. Three-dimensional models for the distribution of mass in galaxies. Publ. Astron. Soc. Japan 27: 533-543.

[11] Monet, D.G., D.O. Richstone \& P.L. Schechter. 1981. The effects of massive disks on bulge isophotes. Astrophys. J. 245: 454-458.

[12] Toomre, A. 1982. Some flattened isothermal models of galaxies. Astrophys. J. 259: 535-543.

[13] Johnston, K.V., D.N. Spergel \& L. Hernquist. 1995. The disruption of the Sagittarius dwarf galaxy. Astrophys. J. 451: 598-606. 
[14] Patsis, P.A., E. Athanassoula, P. Grosbøl, \& Ch. Skokos. 2002. Edge-on boxy profiles in non-barred galaxies. Mon. Not. R. Astron. Soc. 335: 1049-1053.

[15] Helmi, A. \& S.D.M. White. 1999. Building up the stellar halo of the Galaxy. Mon. Not. R. Astron. Soc. 307: 495-517.

[16] Kuzmin, G.G. 1956. Model of the steady Galaxy allowing of the triaxial distribution of velocities. Astron.Zh. 33: 27-45.

[17] Binney, J. \& S. Tremaine. 1987. Galactic Dynamics. Princeton University Press, Princeton, N.J.

[18] de Zeeuw, P.T. 1985. Elliptical galaxies with separable potentials. Mon. Not. R. Astron. Soc. 216: 273-334.

[19] Lees, J.F. \& M. Schwarzschild. 1992. The orbital structure of galactic halos. Astrophys. J. 384: 491-501.

[20] Terzić, B. 1998. Irregular period-tripling bifurcations in axisymmetric scalefree potentials. Ann. New York Acad. Sci. 867: 85-92.

[21] Plummer, H.C. 1911. On the problem of distribution in globular star clusters. Mon. Not. R. Astron. Soc. 71: 460-470.

[22] Richstone, D.O. 1980. Scale-free axisymmetric galaxy models with little angular momentum. Astrophys. J. 238: 103-109.

[23] Helmi, A. 2004. Is the dark halo of our Galaxy spherical? Mon. Not. R. Astron. Soc. 351: $643-648$.

[24] Helmi, A. 2004. Velocity trends in the debris of Sagittarius and the shape of the dark matter halo of our Galaxy. Astrophys. J. 610: L97-100.

[25] Evans, N.W. 1994. The power law galaxies. Mon. Not. R. Astron. Soc. 267: 333-360.

[26] Wisdom, J. 1982. The origin of the Kirkwood gaps: a mapping for asteroidal motion near the 3/1 commensurability. Astron. J. 87: 577-593.

[27] Aguilar, L., P. Hut \& J.P. Ostriker. 1988. On the evolution of globular cluster systems. I. Present characteristics and rate of destruction in our Galaxy. Astrophys. J. 335: 720-747. 